\title{
The Research of Railway Transportation Business Intelligence Based on Ontology
}

\author{
Shangyan Jiang ${ }^{a}$, Lei Huang ${ }^{b}$ \\ School of Economics and Management, Beijing Jiaotong University, Beijing 100044, China. \\ ajiangshangyan414@sina.com, blhuang@bjtu.edu.cn
}

\begin{abstract}
In the 21st century, the world's rapid economic growth brings with it an increase in railway transportation throughput continued year after year, in order to improve the quality of its railway enterprise information management efficiency, business intelligence technology for enterprise operations management, according to the enterprise railway construction scheme proposed business intelligence platform internal management needs. With this business intelligence platform, organizations can make decisions faster, better filtering large amounts of data to improve business operations of enterprises, and ultimately increase the professional level. The role of the ontology is to capture knowledge in related fields and achieves a common understanding of knowledge in the field, make sure all words are commonly recognized in this area, given the relationship between these words from the various levels of formal models clearly defined. The physical structure of a good description of complex data, the use of specialty ontology knowledge representation and reasoning, and build ontology railway business areas. Qualitative knowledge ontology library to implement business intelligence systems reasoning. This paper analyzes the research status and problems of business intelligence and ontology, and after in-depth study of the body and the theory of business intelligence, ontology technology into the railway transportation business intelligence. The actual research in the field of railway transportation business to tease out the business processes can be achieved, given ontology technology areas of business building process.
\end{abstract}

Keywords: Business intelligence system; Ontology; Intelligent decision; Railway.

\section{Introduction}

The business department of the railway enterprise is directly facing the customer department, also is an important part of railway enterprise information. In the railway transportation business sector, how to better interpret and analyze the business-related vocabulary, how better apply to the railway transportation business decision-making, get more and more people's attention. With the development of economy, the railway of the rising volume of business in the world. In improving the service quality and service level of the railway transportation at the same time, how to improve the decision-making ability of the enterprise is very important.

\section{Topic Background}

\subsection{General Background of Railway Transportation Development}

According to the 2017 Top 50 Global Transportation Providers, China Railway Corporation is the largest rail company by revenue of the world. According to the ranking, before 2016, the latest ranking of the revenue of eight major railway companies in the world is: China Railway Corporation, Russian Railway, United Pacific, Burlington Northern Santa Fe, Indian Railway, CSX, Norfolk Southern, and Canadian National Railway [6]. According to public data, transportation demand for commodities increased due to the good macroeconomic conditions in 2017. Affected by many factors, such as overloading of highway and environmental protection restrictions on automobile coal transportation, the railway freight volume showed a rapid rising trend. In 2017, railway completed 3.689 billion tons of freight, up 11.53 percentage points from 2016 to 10.73 percent [7]. The freight volume of major commodities (coal, iron and steel and non-ferrous metals, metal ores, oil and grain, etc.) accounts for a large proportion of railway freight volume, and coal is the most important product. The development of railway freight will play a bigger role in the economic development of our country. 


\subsection{Practical Research Background}

In 21 st century rapid economic development today, the railway transportation needs to face the rapid expansion of business volume, increasing the risk of financial goods, customer personalized requirements increasing these potential problems. As an important means of management reform, information technology has been paid more and more attention in railway enterprises. It can quickly improve the management level, optimize enterprise decision-making, complete the real-time monitoring and accurate reflection of the data, and ultimately improve the efficiency of enterprises.

Railway transportation business management model includes: contract management and document management, billing management, financial management. This model is the railway enterprise to attract customers, gain profit, but also the key to the implementation of relevant policies. Railway business management directly contact external customers, partners and internal production operations. The railway transportation commerce department has gathered the railway enterprise finance, the customer, the service and so on massive data, is the railway enterprise overall management and the policy-making important basis.

\subsection{Research Status of Ontology Technology}

There are many research projects on ontology in foreign countries, and the research results are rich, and a lot of ontology repositories that have already been put into use. There are few literatures about ontology library at home and abroad. The mature ontology library attracts: WordNet, DBpedia, Cyc, HowNet and two domain ontology.

\section{Theoretical Research on Ontology}

\subsection{Definition of Ontology}

Ontology is the first category of philosophy, along with the continuous development of artificial intelligence, ontology has also been given a new meaning. These new meaning is also in constant development and change as shown in Table 1;

Table 1. Ontology definition

\begin{tabular}{|c|c|c|}
\hline category & $\begin{array}{c}\text { Put-forward time } / \\
\text { person }\end{array}$ & definition \\
\hline philosophy & $\begin{array}{c}17 \text { th } \\
\text { Century/Goclenius }\end{array}$ & $\begin{array}{c}\text { A systematic explanation and explanation, it is objective existence } \\
\text { also can reflect objective fact. [1] }\end{array}$ \\
\hline \multirow{3}{*}{$\begin{array}{c}\text { computer } \\
\text { science }\end{array}$} & $1991 /$ Neches & $\begin{array}{c}\text { The basic terms and relationships that constitute the vocabulary of } \\
\text { related fields are given. And the use of these terms and } \\
\text { relationships constitute the definition of the rules of these lexical } \\
\text { extensions [2] }\end{array}$ \\
\cline { 2 - 3 } & $1993 /$ Gruber & Clear specification of the concept system [3] \\
\cline { 2 - 3 } & $1997 /$ Borst & Formal specification of sharable conceptual systems. [4]
\end{tabular}

\subsection{Ontology Modeling}

In practical applications, it is not necessary to construct ontology strictly according to the above 5 kinds of meta languages. At the same time, the relationship between concepts is not limited to the 4 basic relationships listed in Table 2 . The corresponding relationship can be defined according to the specific circumstances of a specific field to meet the needs of application. 
Table 2. Four basic relations of Ontology

\begin{tabular}{|c|c|}
\hline Relationname & Relation description \\
\hline part-of & Relationship between part and whole of concept \\
\hline kind-of & The inheritance relationship between concepts \\
\hline instance-of & Relationship between concept instance and concept \\
\hline attribute-of & A concept is another attribute of a concept \\
\hline
\end{tabular}

\section{The Characteristics of Business Intelligence}

Business intelligence is a subject oriented, integrated, non-renewable (stability), changing with time (time) collection of data in support of management's decision-making process, business intelligence project has the following characteristics:

\subsection{Business Intelligence Projects in Foreign High Failure Rate}

Integrators lack of core business support data and business in-depth understanding of the pursuit of large and complete from the beginning, the rough business and customer information granularity, the initial investment is estimated that the lack of immediate quantitative decision-making application of return on investment ROI, the demand for secondary development is estimated, the integrity and accuracy of the data source is not high. The reason for these management and technology is that the complexity of business intelligence projects increases and the risk of the project increases.

\subsection{Business Intelligence Project Objectives are Diverse}

For a single project, the general goal is single and relatively simple; For business intelligence projects, the use of different business people has different requirements, senior management, business backbone and professional analysts, because the nature of each job has a different goal and different characteristics, which decided the diversity of business intelligence projects. But also because of the diversity of goals, which also led to the management of the difficulty and complexity will be greatly increased.

\subsection{Information Complexity}

Business intelligence project information processing is very complex. From the internal environment, there are business intelligence between the sub-projects, as well as the project and the relevant functions of the company's communication between the information. From the external environment, there are information exchange between the project team and the user industry standard, the exchange of information at the provincial level, and the communication with the user level of the city company, especially the provincial and municipal companies, including the multiple parts communication as well as communication with other third parties, such as the original vendor, developers and integrators that provide other system interfaces. In addition, the channels and methods of information transmission are diverse, including telephone fax, written, E-MAIL, website, conference, presentation and so on.

\section{Realization of Railway Transportation Business Intelligence System Based on Ontology}

\subsection{Construction Methods}

Now the ontology construction method is not the authority of the authority of the certification method. At present, ontology construction has not yet formed an engineering activity and there is a lack of ontology modeling standards and guiding principles. But with the continuous exploration of researchers, has been formed and have a certain impact ontology construction method are: KBSI development IDEF5 method, University of Edinburgh MIke Ushold and Michael Gruninger, who developed the Skeletal Methodology (skeleton method). The methodology of artificial intelligence 
laboratories at the Madrid University of Madrid, University of Toronto Michael Gruninger and Mark. S Fox and others from the TOVE project summed up the TOVE, KACTUS engineering methods [11].

\subsection{Select Modeling Language: OWL}

OWL is the W3C summary of several language development experience and was formally launched in February 2004. It is developed by the W3C for semantic ontology language, using DAML + OIL as a starting point, designed for all aspects of demand. It preserves the compatibility of RDFS, DAML and OIL, and ensures a powerful semantic expression and descriptive logic. Some of the features of OWL distinguish it from traditional description logic and are more suitable for semantic Web requirements. Specific performance in the following five areas [10].

OWL uses the URI naming mechanism.

OWL uses RDF data types and XML Schema data types as data types.

OWL takes RDF/XML as grammar.

OWL can use annotations, although comments do not represent knowledge, but can be easily read.

OWL can use ontology attributes to explain the ontology version, import already constructed ontology, which supports the sharing and reuse of ontology.

There are many kinds of ontology development tools, including KAON, OilEd, OntoEdit, Ontolingua, Ontosaurus, Cyc, WebOnto and protege [8].

\subsection{Protege}

The interface style protege [9] and common Windows application style. Learning to use it easier. The body structure in Protégé is displayed in a hierarchical directory structure. Users can click on the corresponding items to add or edit classes, subclasses, attributes, instances, etc., so that users can design the domain model at the concept level, without the need to understand the specific ontology language. In addition, Protégé for Chinese users, the most valuable is that it supports Chinese.

The most important advantage of protege architecture is its open modular style and componentbased architecture enables system developers to add new functionality by generating appropriate plug-ins.

\section{Construction of Railway Transportation Business Intelligence}

Because of the high level of informatization, the railway business departments generally accept the concept of ontology, and because of the long working system switching frequently, eagerly looking forward to a unified system and unified process. Most of the business people have experienced railway enterprise software development changes, so they generally believe that the early development of business application systems is still in use, and some are the main business support software. Some of the early development language and the corresponding development platform are no longer supported by the current equipment, only by a small number of older generation developers to maintain, there is a considerable application risk. In the railway business department, the business process is clearer, and at the same time, various specifications and rules are quite detailed, so ontology is easier to use.

Using protege, we can make a sketch of the ontology of railway business department in Fig. 1. 


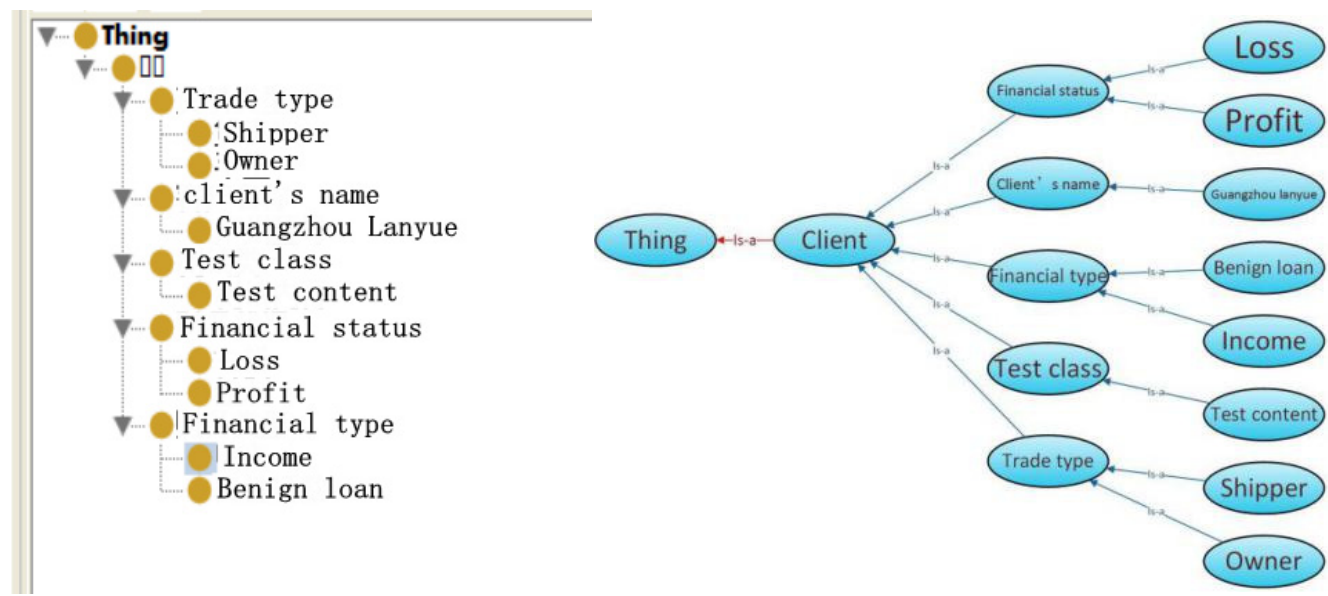

Fig. 1 Schematic diagram of Ontology

Furthermore, we can make further research through ontology diagrams to study the industry, produce and sell vocabulary in Fig. 2.

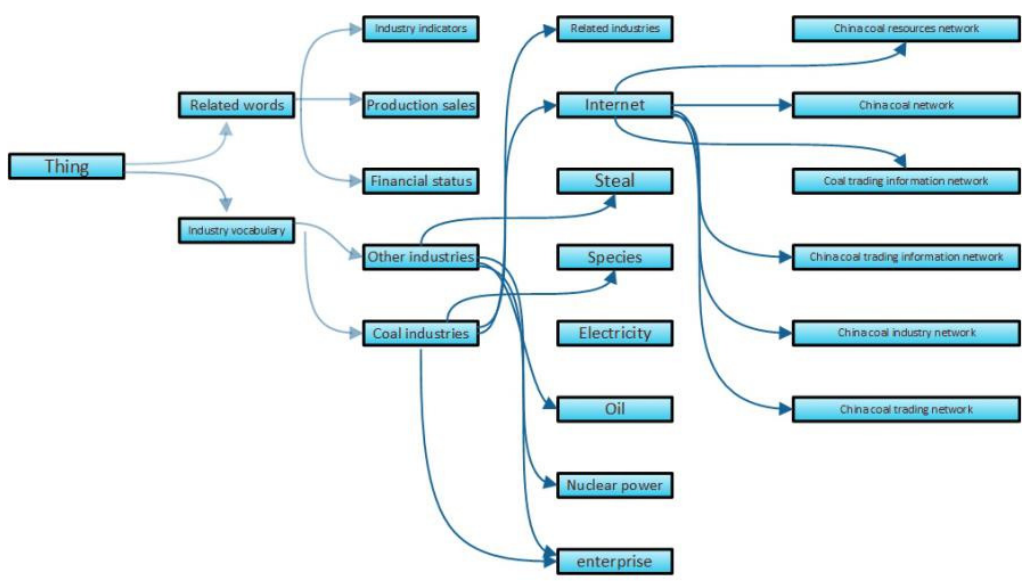

Fig. 2 Further schematic diagram of Ontology

These schematic diagrams are examples of the application of ontology in railway business department. This proves that the ontology construction method can establish the relationship between words well. It provides a direct and sufficient basis for the construction of railway transportation business intelligence.

After introducing ontology, business intelligence is introduced. Business departments can query data from multiple angles and dimensions to meet customer and leader query requirements. At the same time, a variety of statistical data can also be easily generated. Moreover, the introduction of business intelligence enables leaders to make decisions based on historical data and make decisions more scientific. To make the business activities more targeted, which can enhance the railway business department as a whole competitive advantage.

\section{Conclusion}

This article on the ontology and ontology technology in the railway business department of the application of a profound discussion. It mainly includes the construction method of ontology, the choice of ontology modeling language, modeling tools and application of ontology, and the article finally expresses the intelligent business system based on ontology. At the same time, this paper analyzes the business process of railway enterprises and provides theoretical support for constructing railway business ontology through simple ontology model.

The main achievements of this paper are: 
This paper analyzes the operation process of the railway business department in detailed and analyzes the existing problems of the railway business department.

On the basis of the current mainstream ontology model, this paper puts forward the ontology modeling of the railway business department and puts forward the model, combined with the present situation of the railway business department.

At the end of this paper, the necessity and feasibility of intelligent business system based on ontology technology and business intelligence are expounded. The establishment of intelligent business system of railway business department will further promote the development of railway transportation information.

In this paper, we should pay attention to the theory and practice, pay attention to the feasibility and necessity of the theory.

\section{References}

[1]. Liddell, Henry George; Scott, Robert; A Greek-English Lexicon at the Perseus Project.

[2]. NechesR, FikesRE, GruberTR, etal. Enabling Technology for Knowledge Sharing. AIMagazine, 1991, 12(3):36-56.

[3]. Gruber T R. A Translation Approach to Portable Ontology Specifications. Knowledge Acquisition, 1993, 5:199-220.

[4]. Borst W N. Construction of Engineering Ontologies for Knowledge Sharing and Reuse. PhDthesis, University of Twente, Enschede, 1997.

[5]. Studer R, Benjamins V R, Fensel D. Knowledge Engineering, Principles and Methods. Data and Knowledge Engineering, 1998,25(122):161-197.

[6]. Information on: https://www.joc.com/international-logistics/logistics-providers/parcel-carriersfare-best-top-50-revenue-growth_20171029.html.

[7]. Information on: https://www.chyxx.com/industry/201805/640179.html.

[8]. WC. OWL Web Ontology Language[J]. Iee International Workshop on Intelligent Environments. iee. available, 2009, 21(4):125 - 132.

[9]. Knublauch H, Fergerson R W, Noy N F, et al. The Protégé OWL Plugin: An Open Development Environment for Semantic Web Applications [M]// The Semantic Web - ISWC 2004. Springer Berlin Heidelberg, 2004:229--243.

[10]. Hou Mian, Liao Lejian. Implementation of inference engine based on semantic Web ontology language [J]. dual-use technology and products, 2005, (7). DOI: 10.3969/j.issn.10098119.2005.07.017.

[11]. Zhu Jiang, Zhang Cui, Ma Wen. Hybrid ontology method based on Ontology cloud shadow model [J]. Journal of Nanjing University: Natural Science Edition, 2010, 46(5):535-541. 